\title{
LARYNX MYOFIBROBLASTIC TUMOR, A RARE CASE REPORT
}

\author{
Borja Bazán Inostroza ${ }^{1}$, Jorge Prada Pendolero ${ }^{1}$, Gustavo Eisenberg Plaza ${ }^{1}$, Eduardo \\ Raboso García-Baquero ${ }^{1}$, and Maria Magdalena Adrados ${ }^{1}$ \\ ${ }^{1}$ Hospital Universitario de la Princesa
}

December 24, 2021

\begin{abstract}
Myofibroblastic tumour is extremely rare in the larynx, with just over 40 published cases. Despite being a benign tumour, they are tumours with a marked inflammatory character, local destruction and the possibility of degeneration to malignant histological types and with metastatic capacity. Anatomopathological differential diagnosis is fundamental in this cases.
\end{abstract}

\section{INTRODUCTION:}

Myofibroblastic tumours of the larynx are a very rare finding with approximately 40 cases published in the scientific literature (1). They are benign neoplasms with a marked inflammatory character that affect soft tissues, can become locally invasive, malignant or metastatic. In most cases they are asymptomatic spaceoccupying lesions, which require differential diagnosis with other laryngeal neoplasms. There are several nomenclatures to define this entity, but we will use the term inflammatory myofibroblastic tumour described by Umiker in 1954, as it is the most commonly used name and was designated as the official nomenclature by the World Health Organisation in 1994 (2). The term inflammatory alludes to the benign nature of this neoplasm; however, cases of advanced local invasion have been described due to the inflammatory nature of the lesion itself and degeneration to more aggressive histological types in the larynx. The most frequently described locations of this tumour are the lungs, lymph nodes, spleen, liver, mesentery and wall of the gastrointestinal tract (2).

They can also affect the breast, bones, nerves and central nervous system (4) (5).

In the head and neck area they are infrequent, with described involvement of parapharyngeal spaces, oropharyngeal, nasopharyngeal, pterygopalatine fossa, infratemporal fossa, paranasal sinuses, orbit and oral cavity $(6)(7)(8)(5)$. In the larynx, they mainly affect the vocal cords, with cases described at the supraglottic and subglottic levels (1)(10).

The pathogenesis of this entity is related to reactive processes, infections, autoimmune diseases, laryngeal trauma and other neoplasms, with no clear aetiology described (11)(7). It affects mostly children and young adults (4).

Most cases described at the laryngeal level are asymptomatic. They may cause dysphonia, dyspnoea, pharyngolaryngeal foreign body sensation or dysphagia, among others (13).

\section{METHOD:}

We present the case of a 39-year-old man who had been seen at another centre for clinical symptoms of dysphonia that had been evolving for months. He was referred for pathological findings of an inflammatory 
myofibroblastic tumour of the right vocal cord after biopsy by direct laryngoscopy. The patient had no personal history of interest or toxic habits, he was not a smoker or drinker and did not report any consumption of illicit substances. The blood count and coagulation were normal, with the only abnormal finding in the biochemical study being LDL cholesterol $280 \mathrm{mg} / \mathrm{dl}$. During the examination, the patient presented symptoms of dysphonia and pharyngolaryngeal foreign body sensation of months of evolution. The fibrolaryngoscopic examination revealed a lesion with the appearance of a pedunculated angiomatous polyp $<1 \mathrm{~cm}$ in its longest axis, in the anterior third of the right vocal fold, without involvement of the anterior commissure (Image 1). The rest of the physical examination was normal, and no cervical lymphadenopathy was observed during the cervical examination.

\section{RESULTS:}

Surgical resection was scheduled by the Otorhinolaryngology Department. Partial cordectomy was performed with $\mathrm{CO} 2$ laser with complete resection of the lesion with margins up to the muscular plane of the vocal cord, respecting the anterior commissure. The anatomopathological result of the surgical specimen was focal mesenchymal proliferation with ALK expression compatible with myofibroblastic inflammatory tumour (Image 2 and 3). The case was presented to the Head and Neck Tumour Committee of the hospital, where it was decided to follow it up by the Otorhinolaryngology Department. During the following months, an area of surgical sequelae was observed in the area of partial cordectomy of the right vocal cord with marked fibrosis and granulation, which acquired a nodular appearance over the months of follow-up. After 6 months of followup, it was decided to perform microsurgery on the larynx to take a biopsy to rule out recurrence (Image 4). Laryngeal microsurgery was scheduled, where biopsies were obtained from the nodular fibrotic area with suspected recurrence. The pathological findings of the biopsies showed respiratory mucosa, granulation tissue and chronic inflammatory changes of a non-specific nature. After 8 months of follow-up, there was no evidence of recurrence. Given the risk of recurrence and metastasis of this pathology, the Committee decided to continue close follow-up for at least 12 months as is done in most series published in the literature.

\section{DISCUSION:}

Head and neck cancer comprises malignant lesions affecting the oral cavity, pharynx, larynx, salivary glands, as well as the nasal cavity and paranasal sinuses. Worldwide, it ranks seventh among malignant neoplasms. In the USA, there are more than 54,000 new cases per year with an incidence of around 15/100,000 inhabitants and 12,000 deaths attributable to this disease (14). According to data from the Spanish Society of Otorhinolaryngology and Head and Neck Surgery (SEORL-CCC), about 10,000 new cases are detected each year in Spain (15), mainly in men between 45 and 65 years of age. The most common histology is epidermoid carcinoma, which is related to alcohol and tobacco consumption. Laryngeal tumours with histology other than squamous cell carcinoma are rare with an incidence of $<1 \%$. Most of these are mesenchymal tumours (10). Inflammatory myofibroblastic tumour (IMT) is an extremely rare subtype in the larynx but more frequent in other areas of the head and neck. Clinically it mimics other more common laryngeal neoplasms, requiring a differential diagnosis.

Clinically they behave as asymptomatic masses or may cause symptoms of a space-occupying lesion, causing dysphonia, dyspnoea, dysphagia or pharyngeal-laryngeal foreign body sensation. Locally, the inflammatory and expansive behaviour of the lesion can cause great clinical variability, mimicking other neoplastic processes. Primary involvement at the laryngeal level with systemic symptoms including constitutional syndrome, fever, anaemia or thrombocytopenia has been described (16)(17), which reflects the variability and morbidity associated with this pathology. The definitive diagnosis is eminently anatomopathological.

Histologically is composed of lymphocytes, plasma cells, histiocytes, fibroblasts and myofibroblasts in variable proportion, forming four basic histological patterns: predominant lymphoplasmocytic infiltrate, predominant lymphohistiocytic infiltrate, myofibroblastic infiltrate and lymphocytic infiltrate with collagen deposition (3).

Immunohistochemistry is characteristic of a soft tissue tumour with ALK cytoplasmic positivity in most cases, without being a specific finding for this type of tumour. Variable cytoplasmic positivity for vimentin, smooth muscle actin, muscle specific actin and desmin, and negativity for myoglobin, myogenin and S100 
protein is characteristic $(2,18)$.

Characteristic is the finding of genetic rearrangements on chromosome $2 \mathrm{p} 23$ with activation of the ALK receptor tyrosine kinase gene leading to overexpression and activation of the ALK protein, without being a specific finding, which occurs in approximately $50 \%$ of cases and appears to have an age-dependent distribution, with this finding being more common in adults under 40 years of age. This overexpression also appears to have a morphological distribution within the tumour itself, with activation occurring in the myofibroblastic component without expression of this genetic alteration in the inflammatory cells of the tumour itself. In any case, if there is no genetic alteration in ALK, genetic rearrangements have been observed in the HMGIC gene (also known as HMGA2) on chromosome 12, $\mathrm{t}(2,17),(\mathrm{p} 23$, q23), tropomyosin 4 (TPM 4), TPM 3, t(p25, p23), cysteinyl tRNA synthetase and Ran binding protein. In addition, TP53 positivity has been demonstrated in some cases, which could have prognostic influence, being more common in recurrent cases, with transformation to malignant histological types or production of metastases $(2,7,13,19)$.

Despite being very infrequent lesions in the larynx, IMT is a pathology to be taken into account, which requires the same management as laryngeal neoplasms, with biopsies taken to establish the definitive anatomopathological diagnosis and define a treatment plan. The first-line treatment is surgery, either with cold instruments or resection with laser surgery, the fundamental prognostic factor being radical excision with free edges. Radiochemotherapy is reserved for cases with positive margins or a formal contraindication to surgery. Close clinical follow-up of this pathology is essential, with most series requiring close follow-up of the patient for at least 12 months, given the variable risk of recurrence and distant metastasis $(7,13,16,17,20,21)$.

Given the marked inflammatory component of the lesion, second-line corticosteroid therapy is validated as an alternative therapy in cases with contraindications for surgery or tumour recurrence and is also used for head and neck IMT in locations other than the larynx (1).

Genetic alterations also influence new lines of treatment, with published series of treatment with the ALK inhibitor crizotinib in cases of ALK-positive ITM as second-line therapy in cases of contraindication to surgery, partial resection or positive surgical margins $(13,22)$.

CONCLUSION:

IMT is an infrequent pathology in the larynx, but it is included in the differential diagnosis of benign laryngeal pathology. Despite this, it is essential to take into account the locally expansive and inflammatory nature of this lesion, the risk of transformation to malignant histological types and the possibility of distant metastasis. Anatomopathological and immunohistochemical study is fundamental in the differential and definitive diagnosis. The first-line treatment is surgical resection, complete with free margins, with close postoperative follow-up given the possibility of local and distant recurrence.

BIBLIOGRAPHY: 